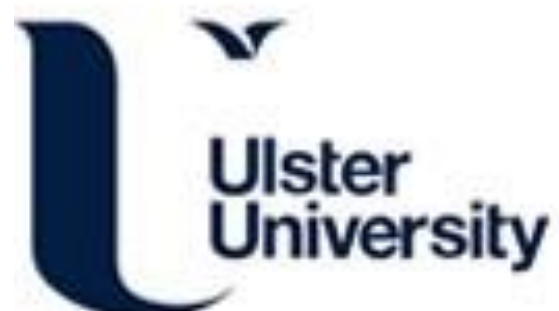

\section{A network analysis of DSM-5 posttraumatic stress disorder and functional impairment in UK treatment-seeking veterans}

Ross, J., Murphy, D., \& Armour, C. (2018). A network analysis of DSM-5 posttraumatic stress disorder and functional impairment in UK treatment-seeking veterans. Journal of Anxiety Disorders, 57, 7-15. https://doi.org/10.1016/j.janxdis.2018.05.007

Link to publication record in Ulster University Research Portal

Published in:

Journal of Anxiety Disorders

Publication Status:

Published (in print/issue): 30/06/2018

DOI:

10.1016/j.janxdis.2018.05.007

\section{Document Version}

Author Accepted version

\section{General rights}

Copyright for the publications made accessible via Ulster University's Research Portal is retained by the author(s) and / or other copyright owners and it is a condition of accessing these publications that users recognise and abide by the legal requirements associated with these rights.

\section{Take down policy}

The Research Portal is Ulster University's institutional repository that provides access to Ulster's research outputs. Every effort has been made to ensure that content in the Research Portal does not infringe any person's rights, or applicable UK laws. If you discover content in the Research Portal that you believe breaches copyright or violates any law, please contact pure-support@ulster.ac.uk. 


\section{Accepted Manuscript}

Title: A network analysis of DSM-5 posttraumatic stress disorder and functional impairment in UK treatment-seeking veterans

Authors: Jana Ross, Dominic Murphy, Cherie Armour

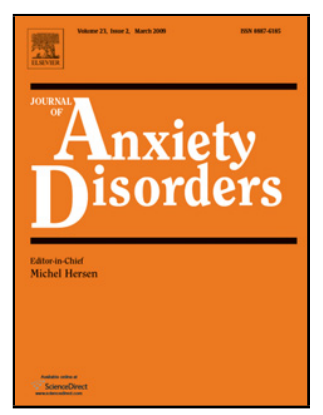

PII:

S0887-6185(18)30072-0

DOI: https://doi.org/10.1016/j.janxdis.2018.05.007

Reference: ANXDIS 2031

To appear in: Journal of Anxiety Disorders

Received date: 23-2-2018

Revised date: $10-5-2018$

Accepted date: 26-5-2018

Please cite this article as: Ross, Jana., Murphy, Dominic., \& Armour, Cherie., A network analysis of DSM-5 posttraumatic stress disorder and functional impairment in UK treatment-seeking veterans.Journal of Anxiety Disorders (2018), https://doi.org/10.1016/j.janxdis.2018.05.007

This is a PDF file of an unedited manuscript that has been accepted for publication. As a service to our customers we are providing this early version of the manuscript. The manuscript will undergo copyediting, typesetting, and review of the resulting proof before it is published in its final form. Please note that during the production process errors may be discovered which could affect the content, and all legal disclaimers that apply to the journal pertain. 
Running head: PTSD and functional impairment

A network analysis of DSM-5 posttraumatic stress disorder and functional impairment in UK treatment-seeking veterans

\author{
Jana Ross, $\mathrm{PhD}^{\mathrm{a}}$ \\ Dominic Murphy, $\mathrm{PhD}^{\mathrm{bc} *}$ \\ Cherie Armour, $\mathrm{PhD}^{\mathrm{a}}$
}

\begin{abstract}
${ }^{a}$ Psychology Research Institute, Faculty of Life \& Health Sciences, Ulster University, Coleraine, Northern Ireland, UK (j.ross@ulster.ac.uk; c.armour1@ulster.ac.uk)

${ }^{\mathrm{b}}$ Research Department, Combat Stress, Leatherhead, UK (dominic.murphy@combatstress.org.uk)
\end{abstract}

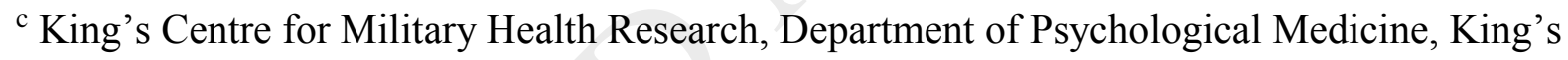
College London, London, UK

* Corresponding author: Dr Dominic Murphy, Research Department, Combat Stress, Tyrwhitt House, Oakland Road, Leatherhead, KT22 0BX, UK. Email: dominic.murphy@combatstress.org.uk

Word count: 6,349 
PTSD and functional impairment

\title{
Highlights
}

- $\quad$ PTSD symptoms B1, B2, D4, D6 and E4 were the most central symptoms

- Functional impairment was related to a variety of PTSD symptoms

- Impaired relationships were associated primarily with numbing symptoms

- Impaired home management was associated primarily with reexperiencing symptoms

\begin{abstract}
Network analysis is a relatively new methodology for studying psychological disorders. It focuses on the associations between individual symptoms which are hypothesized to mutually interact with each other. The current study represents the first network analysis conducted with treatment-seeking military veterans in UK. The study aimed to examine the network structure of posttraumatic stress disorder (PTSD) symptoms and four domains of functional impairment by identifying the most central (i.e., important) symptoms of PTSD and by identifying those symptoms of PTSD that are related to functional impairment. Participants were 331 military veterans with probable PTSD. In the first step, a network of PTSD symptoms based on the PTSD Checklist for DSM-5 was estimated. In the second step, functional impairment items were added to the network. The most central symptoms of PTSD were recurrent thoughts, nightmares, negative emotional state, detachment and exaggerated startle response. Functional impairment was related to a number of different PTSD symptoms. Impairments in close relationships were associated primarily with the negative alterations in cognitions and mood symptoms and impairments in home management were associated primarily with the reexperiencing symptoms. The results are discussed in relation to previous PTSD network studies and include implications for clinical practice.
\end{abstract}


PTSD and functional impairment

Keywords: PTSD; Functional impairment; DSM-5; Network analysis; Veterans; Treatmentseeking 
PTSD and functional impairment

\section{Introduction}

Posttraumatic stress disorder (PTSD) has been widely discussed in relation to military veterans' mental health (e.g., Benyamini \& Solomon, 2005; Wisco et al., 2014). It is a heterogeneous disorder characterized by symptoms of reexperiencing, avoidance, negative alterations in cognitions and mood and hyperarousal (American Psychiatric Association [APA], 2013). PTSD can have significant negative effects on service leavers' community reintegration (Karstoft, Armour, Andersen, Bertelsen, \& Madsen, 2015; Sayer et al., 2015) and if left untreated, it can impact negatively upon one's overall quality of life, functioning, life satisfaction and general wellbeing (Dobie et al., 2004; Pittman, Goldsmith, Lemmer, Kilmer, \& Baker, 2012; Schnurr, Lunney, Bovin, \& Marx, 2009).

Research has shown that PTSD is strongly associated with impairments in various domains of functioning, including intimate relationships (Taft, Watkins, Stafford, Street, \& Monson, 2011), occupation (Smith, Schnurr, \&Rosenheck, 2005), parenting (Gewirtz, Polusny, DeGarmo, Khaylis, \& Erbes, 2010) and others. This has also been reflected in the diagnostic criteria for PTSD according to the fifth edition of the Diagnostic and Statistical Manual of Mental Disorders (DSM-5; APA, 2013), which require significant impairment in one or more areas of functioning before a diagnosis of PTSD can be made. A related area of research has focused on identifying specific PTSD symptom clusters (e.g., re-experiencing symptom cluster, avoidance symptom cluster) that have the strongest relationship with different areas of functioning. Such research is important, considering that not all PTSD symptoms are created equal. This has been directly demonstrated in studies which have found differential associations of the PTSD symptom clusters with a variety of external constructs (e.g., Pietrzak et al., 2015; Roley, Contractor, Weiss, Armour, \& Elhai, 2017). In relation to functional impairment, studies have shown that the most consistent predictor of various domains of functioning is the PTSD emotional numbing symptom cluster. For example, 
PTSD and functional impairment

Schnurr and Lunney (2008) reported that in a sample of 358 male and 203 female veterans with current PTSD, emotional numbing was the only symptom cluster associated with the quality of life, after controlling for the effects of other PTSD symptom clusters and demographic covariates. The prominent role of emotional numbing in functional impairment has also been reported in other studies with different samples (Kuhn, Blanchard, \& Hickling, 2003; North et al., 1999; Pietrzak et al., 2015; Rona et al., 2009; Ruscio, Weathers, King, \& King, 2002; Shea, Vujanovic, Mansfield, Sevin, \& Liu, 2010).

In recent years, studies utilizing network analysis to look at the relationships between PTSD symptoms and between PTSD symptoms and external constructs started to emerge (cf. Armour, Fried, \& Olff, 2017). A network consists of variables that are hypothesized to interact and exert potentially causal effects upon each other (Borsboom \& Cramer, 2013). Because of the differential interactions, the variables, usually symptoms of a specific disorder, such as PTSD, are not considered to be interchangeable. Central symptoms are likely to be more influential than others (i.e., peripheral symptoms) and once activated, these central symptoms will rapidly spread the activation throughout the network, thus giving rise to other PTSD symptoms (Borsboom \& Cramer, 2013). These predictive relationships need to be tested with time-series data, however, cross-sectional networks may generate important exploratory insights into the predictive effects of different variables (Epskamp, Waldorp, Mõttus, \& Borsboom, 2018). It has also been suggested that central symptoms could be viable targets for interventions (Armour, Fried, Deserno, Tsai, \& Pietrzak, 2017; van Borkulo et al., 2015), because theoretically, eliminating a central symptom should decrease the activation of the connected symptoms, thus speeding up recovery. This is especially true in person-specific networks (Borsboom \& Cramer, 2013), but again, it should be tested with time-series data. 
PTSD and functional impairment

Previous PTSD studies conducted in this area have identified different central symptoms, although the least central symptom identified in PTSD network studies has almost consistently been memory impairments (e.g., Armour et al., 2017; Spiller et al., 2017), defined as the "inability to remember an important aspect of the traumatic event(s)" (APA, 2013, p. 272). Such findings support the results from factor analytic literature (e.g., Forbes et al., 2015; Yufik \& Simms, 2010), which has suggested that memory impairments may be the least important symptom of PTSD.

Several network analytic studies conducted in the area of PTSD have also examined the relationships between specific PTSD symptoms and external correlates. By entering other variables into the PTSD network, it becomes possible to identify those symptoms of PTSD that are most strongly related to these external psychosocial constructs. Studies have looked at the relationship of PTSD symptoms with depression (Afzali, Sunderland, Teesson et al., 2017; Choi, Batchelder, Ehlinger, Safren, \& O’Cleirigh, 2017), alcohol use disorder (Afzali, Sunderland, Batterham et al. 2017), sexual risk behaviour (Choi et al., 2017) and a mixture of different constructs (Armour et al., 2017; Birkeland \& Heir, 2017). No studies have, however, looked at the relationships of PTSD symptoms with impairments in different domains of non-health-related functioning, specifically home management, close relationships and social and private leisure activities.

The aim of the current study was to examine the network structure of PTSD symptoms and functional impairment in UK treatment-seeking military veterans with probable PTSD. So far, there have only been three PTSD network studies conducted with veteran populations and these were all conducted with US veterans (Armour et al., 2017; Mitchell et al., 2017; von Stockert, Fried, Armour, \& Pietrzak, 2018). Moreover, to the best of our knowledge, there have been no network studies which included in their analyses only those individuals who met the diagnostic criteria for probable PTSD. By examining the 
PTSD and functional impairment

network structure of PTSD and functional impairment in a sample of UK military veterans meeting the DSM-5 diagnostic criteria for probable PTSD, the current study specifically aimed to 1) identify the most central PTSD symptoms; and to 2) identify those PTSD symptoms that are related to different domains of functional impairment. The DSM-5 criteria for PTSD require an individual to report "clinically significant distress or impairment in social, occupational, or other important areas of functioning" as a consequence of the symptoms (APA, 2013, p. 272). It is, however, not clear, if any of the PTSD symptoms are associated with more functional impairment than others and this is what the current study aimed to examine.

\section{Methods}

\subsection{Participants and procedure}

This study utilised data from a cross-sectional dataset of a nationally representative sample of UK treatment-seeking veterans with mental health difficulties (Murphy, Ashwick, Palmer, \& Busuttil, 2017). The sample was recruited from a national veteran-specific mental health charity called Combat Stress (CS), which provides clinical mental health services across the UK. Between January $31^{\text {st }}, 2015$ and February $1^{\text {st }}$, 2016, 3,335 unique individuals had attended at least one appointment with CS. From this population, a 20\% subsample of 667 individuals was randomly drawn. Sixty-seven of these individuals were removed from the sample because four had died prior to data collection commencing and 63 were later removed because of insufficient address information. The final sample size was 600 . Individuals were sent questionnaires over three mail-out waves and then telephone tracing was employed to elicit responses. A total of $67.2 \%$ (403/600) returned completed questionnaires. 
PTSD and functional impairment

For the current study, participants were restricted to only individuals who meet the case criterion for probable PTSD on the PTSD Checklist for DSM-5 (PCL-5). A cut-off of 34 or above on the PCL-5 was used to indicate probable PTSD. This cut-off had previously been validated against the Clinician Administered PTSD Scale for DSM-5 within this population (Murphy, Ross, Ashwick, Armour, \& Busuttil, 2017). This restricted the effective sample size for the current study to 331 participants.

\subsection{Measures}

\subsubsection{PTSD Checklist for DSM-5 (PCL-5)}

The PCL-5 (Weathers et al., 2013) is a self-report measure assessing the severity of 20 PTSD symptoms arising in response to each participant's traumatic experiences. The 20 items assess the four DSM-5 PTSD symptom clusters of reexperiencing, avoidance, negative alterations in cognitions and mood, and hyperarousal. Participants indicate the extent to which each symptom bothered them over the past month, using a five-point Likert scale ranging from $0=$ Not at all to $4=$ Extremely. Higher scores indicate greater PTSD symptom severity. Cronbach's alpha in the current sample was .839 .

\subsubsection{Work and Social Adjustment Scale (WSAS)}

The WSAS (Marks, 1986) is a five-item self-report measure assessing five domains of functional impairment; Ability to work, Home management, Social leisure activities, Private leisure activities, and Close relationships. Participants indicate how much their 'problem' affects their functioning in relation to the above domains, using a nine-point Likert scale ranging from $0=$ Not at all to $8=$ Very severely. Higher scores indicate greater impairment. In the current study, the Ability to work domain was not assessed, because some participants were not employed, in which case a skip function was utilized. Using data that relies on skip 
PTSD and functional impairment

functions can lead to inaccurate results as it distorts the correlation matrix used in network estimation (Borsboom et al., 2017). Cronbach's alpha for the four items utilized in the current study was .758.

\subsection{Data analysis}

The primary analysis involved an estimation of a PTSD network consisting of 20 PCL-5 items. The secondary analysis involved the addition of four functional impairment items (WSAS) to the PTSD network (i.e., a total of 24 items).

\subsubsection{Network estimation and visualization}

The networks were estimated using the EstimateNetwork function from the R package bootnet, version 1.0.1 (Epskamp, Borsboom, \& Fried, 2017). The items were treated as ordinal and the networks were therefore estimated based on a Gaussian Graphical Model using a polychoric correlation matrix. The Gaussian Graphical Model consists of nodes, representing symptoms, and edges, representing weighted connections between the nodes. These connections can be interpreted as partial correlations and can range from -1 to 1 . With 20 nodes (PTSD network), there are 190 pairwise associations (i.e., edges) and with 24 nodes (PTSD and functional impairment network) there are 276 pairwise associations to be estimated, which is likely to lead to a discovery of some false positive, or spurious, edges. To reduce this possibility, the graphical lasso regularization technique, which shrinks all edges and constrains the very small ones to zero, was employed (Friedman, Hastie, \& Tibshirani, 2008). The resulting network is more parsimonious and contains only the most relevant and strongest edges. The network was visualized using the Fruchterman-Reingold algorithm (Fruchterman \& Reingold, 1991) in R package qgraph, version 1.4.4 (Epskamp et al., 2012). The algorithm places more important nodes (i.e., nodes with more or stronger connections) in 
PTSD and functional impairment

the centre of the network. In the current study, blue lines between nodes represent positive edges and red lines represent negative edges. The thicker and more saturated the line, the stronger the connection.

\subsubsection{Centrality estimation}

The most central symptoms in the PTSD network were identified using the node strength, closeness and betweenness centrality indices (Opsahl, Agneessens, \& Skvoretz, 2010). Centrality is the measure of node interconnectedness. Node strength is the direct connection of a node to the network, calculated as the sum of absolute weights of all edges of the focal node. Node closeness is the indirect connection of a node to the network; it is the average distance of a node to all other nodes in the network, calculated as the sum of the inverse of the shortest path length from the focal node to all other nodes. Node betweenness also represents node's indirect connection; it is the number of times a node lies in the shortest path between two other nodes. More central nodes have higher centrality values.

Following the example of McNally, Heeren and Robinaugh (2017), we then calculated the correlation between strength centrality and the standard deviation of the items in the network to check whether the conclusions about the "importance" of the individual items can be trusted. Terluin, de Boer and de Vet (2016) demonstrated that connection strength could in fact be affected by differential variability in symptom severity ratings.

For the PTSD and functional impairment network, we estimated bridge centrality values using the R package networktools, version 1.1.1 (Jones, 2018). Bridge centrality looks at the connectivity between two disorders, or in our case, between PTSD and functional impairment. We calculated 1) Bridge strength, which is the sum of absolute values of edges between a node and all other nodes in the second disorder, 2) Bridge closeness, which is the average path length from a node to all other nodes in the second disorder, 3) Bridge 
PTSD and functional impairment

betweenness, which is the number of times a node lies on the shortest path between two other nodes that belong to different disorders, 4) Bridge expected influence (1-step), which is the sum of all edges between a node and all other nodes in the second disorder (taking into account positive and negative edges), and 5) Bridge expected influence (2-step), which is the same as 1-step, but also includes the indirect influence of the node through other nodes.

\subsubsection{Network accuracy, stability and significance testing}

The accuracy and stability of the network was examined using the R package bootnet, version 1.0.1 (Epskamp et al., 2017). First, we bootstrapped (2,500 iterations) the 95\% confidence intervals around the edge weights in order to assess the accuracy of the edge weights. Smaller confidence intervals indicate greater accuracy. Second, we used the casedropping subset bootstrap (2,500 iterations) to examine the stability of the order of the node centrality indices. The results indicate whether the order of the centrality indices stays the same when the network is re-estimated with fewer cases. If the correlation between the original order of centrality indices and the order of centrality indices after cases have been dropped remains high, the centrality indices are considered stable (Epskamp et al., 2017). A correlation stability coefficient (CS-coefficient) has been proposed as a measure for quantifying the stability of the node centrality indices. A recent simulation study has shown that the CS-coefficient should be at least 0.25 and preferably above 0.5 for the centrality indices to be interpretable (Epskamp et al., 2017). Finally, we tested for significant differences between edge weights and node centralities using the bootstrapped difference tests. At the time of writing this paper, there is no way to correct for multiple testing.

\subsubsection{Missing data}


PTSD and functional impairment

There were no missing data on PCL-5 items and only minimal amounts of missing data on the WSAS items. Overall, $0.37 \%$ of all data (i.e., PCL-5 and WSAS) was missing. The PTSD with functional impairment network was estimated using complete pairwise observations (i.e., using all available data).

\section{Results}

\subsection{Sample characteristics}

In the effective sample of 331 participants, 318 (96.07\%) were males and $13(3.93 \%)$ were females. Participants were aged 22 to 86 , with average age of $50.42(S D=12.18)$. A total of $221(66.77 \%)$ reported being in a relationship and $110(33.23 \%)$ were single, divorced, separated or widowed. Ninety-two (27.79\%) participants had a job and the rest were not working, either due to ill health $(n=165,49.85 \%)$ or other reasons $(n=74$, $22.36 \%)$. The average elapsed time since leaving the Armed Forces was 19 years $(S D=$ 12.19), ranging from 0 to 63 years. The mean PCL-5 score was $58.57(S D=10.92)$. The mean scores on the WSAS were $5.72(S D=2.38)$ for the social leisure activities item, 5.29 $(S D=2.26)$ for the close relationships item, $4.87(S D=2.39)$ for the home management item, and $4.75(S D=2.62)$ for the private leisure activities item.

\subsection{PTSD network}

Figure 1 depicts the network of 20 PTSD symptoms. The vast majority of the symptoms were positively connected, and the strongest edges (i.e., regularized partial correlations) were found between symptoms C1-C2 (.59; avoidance of thoughts with avoidance of reminders), E3-E4 (.47; hypervigilance with exaggerated startle response), B1B2 (.46; recurrent thoughts with nightmares), and between D3-D4 (.44; distorted blame with negative emotional state). The correlation matrix with all the regularized partial correlation 
PTSD and functional impairment

coefficients is presented in Table S1. The results of the edge weight bootstrap (Figure S1) show a substantial overlap between the $95 \%$ confidence intervals of the edge weights. However, some of the strongest edges showed non-overlapping confidence intervals with quite a few of the other edges in the network. The bootstrap significance tests (Figure S2) revealed that all four of the above-mentioned edges (C1-C2, E3-E4, B1-B2, and D3-D4) were significantly stronger than all other edges in the network (except for B1-B2 which did not differ significantly from B2-B3). The edges between B1-B4 (recurrent thoughts with psychological cue reactivity), D6-D7 (detachment with restricted affect), D5-D7 (diminished interest with restricted affect), B2-B3 (nightmares with flashbacks) and quite a few others were also significantly different than at least half of the other edges in the network (see Figure S2).

$* * *$ Figure $1 * * *$

The standardized centrality indices for the 20 PTSD symptoms are presented in Figure 2. The results of the associated case-dropping subset bootstrap (Figure S3) suggest that node strength is the most stable centrality measure in the current sample, followed by node closeness. The CS-coefficient values supported these findings; 0.595 for strength, 0.284 for closeness, and 0 for betweenness. The three centrality measures were not highly correlated $\left(r_{s}\right.$ $=.32$ between strength and closeness, $r_{s}=.24$ between strength and betweenness, and $r_{s}=.47$ between closeness and betweenness) and we therefore interpreted node strength as well as node closeness, both of which had CS-coefficient values above the recommended 0.25 cut-off (Epskamp et al., 2017). 
PTSD and functional impairment

The most central nodes, according to the node strength index, were symptoms B1 (recurrent thoughts), B2 (nightmares), D4 (negative emotional state), D6 (detachment) and E4 (exaggerated startle response). These symptoms did not differ significantly from each other on strength centrality (Figure S2), however, they were significantly more central than several of the other symptoms in the network. Based on node strength, symptom D1 (memory impairments) was the least central symptom. It was significantly less central than all other symptoms in the network. The correlation between strength centrality and the standard deviations of the PTSD items was not significant $\left(r_{s}=-0.23, p=.328\right)$, suggesting that the variability in item severity ratings did not affect the conclusions about strength centrality. The standard deviations of the PTSD items are presented in Table S2.

The node closeness centrality was highest for symptoms D5 (diminished interest), D7 (restricted affect), D6 (detachment), C2 (avoidance of reminders) and E4 (exaggerated startle response). These symptoms did not differ from each other or from the other PTSD symptoms on node closeness centrality (Figure S5). The only symptom which showed consistently significantly different closeness centrality was symptom D1 (memory impairments); it was significantly less central than all other symptoms in the network, except for symptom E2 (reckless or self-destructive behaviour).

\subsection{PTSD and functional impairment network}

The visual representation of the combined PTSD (20 symptoms) and functional impairment (4 items) network is presented in Figure 3. The results of the edge weight bootstrap (Figure S6) show that quite a few confidence intervals of the edge weights were overlapping, but some of the strongest edges were significantly stronger than the majority of others edges in the network (Figure S7). These were the same edges as the ones identified in 
PTSD and functional impairment

the PTSD-only network, suggesting that the introduction of the functional impairment items into the PTSD network did not affect the connections between these PTSD symptoms. Table S3 presents the correlation matrix with all the regularized partial correlation coefficients. The four functional impairment items were related to each other (regularized partial correlations $.03-.29)$, but they were also related to some of the PTSD items. Relatively small partial correlations were found between impairment in relationships and PTSD symptoms C2, D2, D6, D7 and E1 (.02 - .12), between impairment in private activities and symptoms B5, D5, D7 and E5 (.02 - .05), between impairment in social activities and symptoms B3, C2 and D5 (-.01 - .05), and between impairment in home management and symptoms B1, B2, B5, D5 and E4 (.03 - .09). These relationships were not significantly different from each other (Figure S7). The relationships appear to be weak, but it needs to be remembered that they are regularized (i.e., shrunk) partial correlations. Node centrality indices are not presented, because we were only interested in the connections between the functional impairment items with specific PTSD symptoms.

Standardized bridge centrality indices are presented in Figure 4. Across all indices, PTSD symptoms D5 (diminished interest) and D6 (detachment), had the highest values, thus exerting the most bridging influence on functional impairment. Impairment in home management and impairment in relationships exerted the most bridging influence on the PTSD symptoms.

\footnotetext{
$* * *$ Figure $3 \& 4 * * *$
}

\section{Discussion}

The current study examined the network structure of PTSD symptoms and different domains of functional impairment in a sample of UK treatment-seeking military veterans. To 
PTSD and functional impairment

the best of our knowledge, this is the first network study of PTSD symptoms conducted with UK veterans and the first study conducted with a clinical sample of participants meeting the DSM-5 diagnostic criteria for probable PTSD.

\subsection{PTSD network}

In the PTSD network, the strongest connections were identified between symptoms $\mathrm{C} 1$ and $\mathrm{C} 2$ (avoidance of thoughts with avoidance of reminders), E3 and E4 (hypervigilance with exaggerated startle response), B1 and B2 (recurrent thoughts with nightmares), and between D3 and D4 (distorted blame with negative emotional state). Generally, the connections appeared to be stronger within the DSM-5 PTSD symptom clusters than between them. Supporting the results of Armour et al. (2017), we found that symptom D1 (memory impairments) had the smallest number of connections, which were also relatively weak. This finding is consistent with the factor analytic literature, in which the memory impairments symptom has been consistently found to load weakly on the emotional numbing (DSM-IV studies) or the negative alterations in cognitions and mood (DSM-5 studies) symptom cluster (e.g., Hafstad, Dyb, Jensen, Steinberg, \& Pynoos, 2014; Price \& van Stolk-Cooke, 2015; Seligowski \& Orcutt, 2016; Yufik \& Simms, 2010).

The low connectivity of the memory impairments symptom was further supported in the centrality analyses, where this symptom was significantly less central than all other PTSD symptoms (except for symptom E2 - reckless or self-destructive behaviour). Similar results have been reported in previous DSM-5 and DSM-IV network studies of PTSD (Armour et al., 2017; Birkeland \& Heir, 2017; McNally et al., 2015; Spiller et al., 2017). Taken together, these results imply that the memory impairments item might not be a core symptom of PTSD. In fact, research has shown that memories for traumatic experiences are at least as clear and detailed as alternative memories and they are even clearer and more vivid in individuals with 
PTSD and functional impairment

PTSD than trauma-exposed individuals without PTSD (Megías, Ryan, Vaquero, \& Frese, 2007; Porter \& Birt, 2001). Instead, it has been suggested that this symptom might be a defining feature of the dissociative subtype of PTSD (Miller et al., 2013; Ross, Baník, Dědová, Mikulášková, \& Armour, 2018), as memory impairments have long been considered to be a symptom of dissociation (Dorahy \& van der Haart, 2015; Lanius, Brand, Vermetten, Frewen, \& Spiegel, 2012).

The most central symptoms, based on strength centrality, identified in the PTSD network were symptoms B1 (recurrent thoughts), B2 (nightmares), D4 (negative emotional state), D6 (detachment) and E4 (exaggerated startle response). These symptoms did not differ significantly from each other on strength centrality, but they were significantly more central than some (although not all) other symptoms in the network. The existing DSM-5 network analytic studies of PTSD symptoms have identified different central symptoms. For example, in a sample of 221 US veterans with at least subthreshold PTSD, drawn from the National Health and Resilience in Veterans Study, the most central symptoms were flashbacks, physiological cue reactivity, negative emotional state and detachment (Armour et al., 2017). In a sample of 1,458 US veterans drawn from the longitudinal national registry of Iraq and Afghanistan Army and Marine Corps veterans, the most central symptoms were recurrent thoughts, nightmares, avoidance of thoughts, avoidance of reminders, negative emotional state and restricted affect (Mitchell et al., 2017). In another network study conducted over two time points with 611 US veterans from the National Health and Resilience in Veterans Study, von Stockert et al. (2018) identified negative emotional state, avoidance of thoughts and reminders, nightmares, detachment, difficulty concentrating, psychological and physiological cue reactivity and restricted affect as the most central symptoms at both time points. In yet another DSM-5 network study, conducted with 151 asylum seekers and 
PTSD and functional impairment

refugees in Switzerland, psychological cue reactivity was identified as the most central symptom (Spiller et al., 2017).

It is possible that the different findings are a result of the differences in traumatic exposure, as this factor has previously been found to affect the presentation of PTSD (Chung \& Breslau, 2008). Nevertheless, it could be argued that if the same symptoms of PTSD keep emerging as most central across different studies that utilized different samples with different traumatic experiences and different PTSD prevalence rates, these symptoms may be of high clinical significance when it comes to designing treatment interventions. The most consistent finding from the current study and the previous DSM-5 network studies of PTSD is the high centrality of the negative emotional state symptom. Interestingly, this symptom does not appear in the proposed diagnostic criteria for PTSD according to the eleventh edition of the International Classification of Diseases (ICD-11; World Health Organization, 2018), yet it appears to be of high significance in the existing network studies in that it has high connectivity with other PTSD symptoms. This means that this symptom is likely to interact with other PTSD symptoms in one of three ways: 1) it predicts other symptoms in the network, 2) it is predicted by other symptoms in the network, or 3) it both predicts and is predicted by other symptoms in the network. Network studies based on cross-sectional data, including the current one, are exploratory in the sense that they provide important insights into the relationships between variables in a network. They can generate hypotheses about potentially causal relationships between symptoms and stimulate time-series data research in which the direction of the relationships can be established. It will be important for future studies to examine the predictive relationship of the negative emotional state symptom with other PTSD symptoms.

The aim of ICD-11 is to include only the core symptoms of PTSD that are unlikely to be reported by individuals with differential diagnoses, such as anxiety and depression, thus 
PTSD and functional impairment

increasing the specificity of the PTSD diagnosis and reducing comorbidity (Maercker et al., 2013). The DSM-5 PTSD symptom of negative emotional state, which the current study and the three previous veteran studies (Armour et al., 2017; Mitchell et al., 2017; von Stockert et al., 2018) identified as one of the most central PTSD symptoms could, however, be considered less specific to PTSD, as it is often reported by individuals with other diagnoses. For example, as highlighted by Afzali, Sunderland, Teesson et al. (2017), feelings of sadness and inappropriate guilt are DSM-5 symptoms of major depressive disorder (MDD), but they are markedly similar to the DSM-5 PTSD symptom D4 - "persistent negative emotional state (e.g., fear, horror, anger, guilt, or shame)" (APA, 2013).

One of the strengths of network analysis is its ability to identify the so-called bridge symptoms, which are hypothesized to drive the comorbidity between disorders (Cramer, Waldorp, van der Maas, \& Borsboom, 2010). The nature of the bridge symptoms between the DSM-5 PTSD and depression is yet to be investigated. However, in a study examining the comorbidity between the DSM-IV PTSD and MDD, Afzali, Sunderland, Teesson et al. (2017) found that feelings of sadness and guilt acted as the bridge symptoms between the two disorders. If future studies reveal that the most central PTSD symptoms (e.g., negative emotional state) are also the bridge symptoms between PTSD and MDD, intervening on these symptoms could possibly alleviate symptoms of both disorders.

\subsection{PTSD and functional impairment network}

When the four functional impairment items were added to the PTSD network, the strongest connections between the pairs of PTSD symptoms identified in the PTSD only network were also the strongest ones in the PTSD and functional impairment network (i.e., C1-C2, E3-E4, B1-B2, D3-D4). Different domains of impairment were associated with different PTSD symptoms. However, because the associations were not significantly different 
PTSD and functional impairment

from each other, possibly due to the small sample size, it is not possible to determine which of the symptoms of PTSD have the strongest associations with functional impairment.

The existing literature suggests that functional impairment is related to PTSD primarily through the emotional numbing symptom cluster (Kuhn et al., 2003; North et al., 1999; Rona et al., 2009; Ruscio et al., 2002; Schnurr \& Lunney, 2008; Shea et al., 2010). In the current study, all four domains of impairment were related to at least one PTSD symptom from the negative alterations in cognitions and mood symptom cluster (i.e., D1 - D7), although other symptoms were also associated with impairment.

Impairments in close relationships were associated primarily with emotional numbing symptoms of PTSD (detachment, negative beliefs, restricted affect), but also with the avoidance of reminders and irritability and anger symptoms. Previous studies with military veterans have similarly reported associations between emotional numbing symptoms and problematic relationships (Galovski \& Lyons, 2004; Lunney \& Schnurr, 2007; Riggs, Byrne, Weathers, \& Litz, 1998; Ruscio et al., 2002). It is easy to see how these symptoms could lead to deterioration of, or the inability to form, close relationships. Feelings of detachment or estrangement (D6), negative beliefs, such as 'no one can be trusted' (D2), and the inability to experience positive emotions, such as loving feelings (D7), could all impede the formation or maintenance of attachment with one's children and intimate partner and undermine their ability to form and maintain meaningful supportive relationships. Moreover, irritability and anger (E1) could add tension to the existing relationships or prevent the formation of new ones, if those around feel like they need to 'walk on eggshells' in a fear of upsetting their parent/partner/friend with PTSD.

Impairments in private leisure activities were associated with diminished interest, physiological cue reactivity, restricted affect and sleeping difficulties symptoms. This is not surprising if one considers these PTSD symptoms together; if the individual is no longer 
PTSD and functional impairment

interested in previously enjoyable activities, they are unable to experience positive feelings, such as happiness or satisfaction, and thus cannot derive enjoyment from engaging in such activities, it is hard to see how these activities would not be impaired. Moreover, insomnia can be a precursor to a number of different problems (Harvey, 2008), so it is actually surprising that sleeping difficulties were only related to impairments in private leisure activities and no other domains of functioning in the current study. As shown below, nightmares were also related to impaired functioning (home management), however, a more detailed analysis utilizing a full established sleep measure would be needed to shed more light on these relationships.

Impairments in social leisure activities were associated with diminished interest, flashbacks and avoidance of reminders symptoms. Less research has been conducted in this area, however, Rodriguez, Holowka and Marx (2012) reported that PTSD avoidance and emotional numbing symptoms can be related to impaired friendships and socializing and veterans reporting these symptoms are often reluctant to engage in previously enjoyable activities with their partners.

Finally, impairments in home management were associated primarily with the reexperiencing symptoms (recurrent thoughts, nightmares, physiological cue reactivity), but also with diminished interest and exaggerated startle response symptoms.

Overall, the four functional impairment items did not cluster with the PTSD items, suggesting that the two are separate constructs. However, there were bridge items that connected PTSD with functional impairment and through which activation is likely to spread from one construct to another. For PTSD, these were the symptoms of diminished interest and detachment, which exerted the most influence onto functional impairment, and from the functional impairment items, these were the impaired home management and impaired relationships items, which exerted the most influence onto PTSD. 
PTSD and functional impairment

\subsection{Limitations}

A few limitations associated with the current study need to be acknowledged. First, we used a predominantly male UK military veteran sample, which reduces the generalizability of the findings to other populations. Second, we included 20 PCL-5 items in the estimation of the PTSD network, which limits the interpretation of the centrality results in the current study in the sense that symptoms not included in the network estimation could actually be the most important symptoms of PTSD. Future studies could include PTSD symptoms from non-DSM-based measures, or associated features, such as dissociative symptoms, as there have been debates about whether these should be considered as core features of PTSD (Dorahy \& van der Hart, 2015). Third, our sample was relatively small as we only included individuals with probable PTSD and the results should therefore be interpreted with caution, especially the associations between the PTSD symptoms and the four domains of functional impairment, as there were only few significant differences in the strengths of these associations. Finally, the data was cross-sectional, and it is therefore not possible to infer the temporal order of the symptoms and functional impairment items. Most conceptualizations of PTSD and functional impairment consider the latter to be a consequence of PTSD. It is, however, possible that the two influence each other. For example, it has been found that pre-deployment functional impairment is a significant predictor of post-deployment mental health in US Army soldiers (Wright, Cabrera, Eckford, Adler, \& Bliese, 2012). In relation to our study, it is possible that the PTSD symptoms of emotional numbing, such as detachment and restricted affect, could lead to impaired social relationships, which could in turn lead to reduced social support. Lack of social support could then exacerbate PTSD symptoms, as it has consistently been found to be a strong predictor of PTSD (Brewin, Andrews, \& Valentine, 2000; Fletcher, Elklit, Shevlin \& Armour, 2017). The 
PTSD and functional impairment

relationships identified in the current study should therefore be interpreted with great caution, as the specific predictive role of the individual symptoms cannot be established from crosssectional data.

\subsection{Implications}

The current study has important implications for both researchers and clinicians. First, we identified the most central symptoms in the PTSD network, which has implications for future intervention studies, as it is possible that intervening directly on these symptoms could speed up recovery by partially breaking down the network of interacting symptoms. The results will, however, need to be replicated with time-series data due to the fact that a symptom could be central, not because it is predicting other symptoms itself, but rather because it is predicted by many other symptoms in the network (Fried et al., 2018). Moreover, as cautioned by Fried et al. (2018), just because a symptom is central, it does not necessarily mean it would be an ideal target for interventions. It is possible that feedback loops exist in our network of PTSD symptoms, leading to the activation of central symptoms as soon as these have been "switched off" in therapy. Again, time-series data would be useful to explore this possibility.

The issue of centrality in relation to interventions needs to be further explored by the implementation of specific targeted symptom-level interventions. Additionally, recent developments in the field have pointed to the usefulness of person-specific network models. For example, as shown by Fisher, Reeves, Lawyer, Medaglia and Rubel (2017), specific symptoms may show causal influences in an aggregated data network, but in some individuals, they may have no predictive influence over any symptoms, which could render generic interventions ineffective. This is particularly relevant to heterogeneous conditions 
PTSD and functional impairment

such as PTSD, for which there are almost countless combinations of symptoms that would qualify for a diagnosis (Galatzer-Levy \& Bryant, 2013).

Interventions could also focus on alleviating those PTSD symptoms that are associated with the most impairment. In the current study, symptoms from all four PTSD symptom clusters were associated with functional impairment. Impaired home management was associated primarily with the re-experiencing symptoms and impairments in close relationships were associated primarily with the numbing symptoms of PTSD. However, many other PTSD symptoms were similarly associated with different domains of functioning. It remains for future research to establish whether any symptoms cause significantly more impairment than others. The current study did not identify any differential relationships, however, it is possible that this was due to our study being under-powered. As suggested above, future studies should also examine the predictive relationships between PTSD symptoms and different domains of functioning using time-series data, as it is possible that the relationships are bi-directional. Indeed, the examination of bridge centrality in the current study showed that the PTSD symptoms exerting the most influence on functional impairment were diminished interest and detachment and the functional impairment items exerting the most influence on PTSD symptoms were impairment in home management and impairment in relationships. These results suggest that the activation from one construct to another is likely to occur through these specific bridge items, which warrants future research in this area.

\subsection{Conclusion}

The current study was the first network analysis of PTSD symptoms conducted with a treatment-seeking sample of UK military veterans who meet the diagnostic criteria for probable PTSD. It was also the first study looking at the network of PTSD symptoms and 
PTSD and functional impairment

four different domains of functional impairment. The most central PTSD symptoms were recurrent thoughts, nightmares, negative emotional state, detachment and exaggerated startle response. Considering these results in the context of previous network analytic studies conducted with veteran samples, negative emotional state appears to be the one symptom that should be focused upon in future intervention studies to see if alleviating this symptom leads to improvements in other symptoms. In relation to the PTSD and functional impairment network, the current study showed that many different symptoms of PTSD were related to different domains of functional impairment. None of the PTSD symptoms in the current study were significantly more strongly related to any domains of functional impairment than others. It is, however, possible that this was due to the small sample size utilized in the current study. Future investigations in this area are warranted, as interventions could target not just the most central symptoms of PTSD, but also those that cause the most impairment, or those through which activation is most likely to spread to different domains of functional impairment. 
PTSD and functional impairment

Declarations of interest: none 
PTSD and functional impairment

\section{References}

Afzali, M. H., Sunderland, M., Batterham, P. J., Carragher, N., Calear, A., \& Slade, T. (2017). Network approach to the symptom-level association between alcohol use disorder and posttraumatic stress disorder. Social Psychiatry and Psychiatric Epidemiology, 52, 329-339. doi: 10.1007/s00127-016-1331-3

Afzali, M. H., Sunderland, M., Teesson, M., Carragher, N., Mills, K., \& Slade, T. (2017). A network approach to the comorbidity between posttraumatic stress disorder and major depressive disorder: The role of overlapping symptoms. Journal of Affective Disorders, 208, 490-496. http://dx.doi.org/10.1016/j.jad.2016.10.037

American Psychiatric Association (2013). Diagnostic and Statistical Manual of Mental Disorders (5th ed.). Washington, DC: Author.

Armour, C., Fried, E. I., Deserno, M. K., Tsai, J., \& Pietrzak, R. H. (2017). A network analysis of DSM-5 posttraumatic stress disorder symptoms and correlates in U.S. military veterans. Journal of Anxiety Disorders, 45, 49-59. http://dx.doi.org/10.1016/j.janxdis.2016.11.008

Armour, C., Fried, E., \& Olff, M., (2017). PTSD symptomics: network analyses in the field of psychotraumatology. European Journal of Psychotraumatology, 8(3) 1398003. http://dx.doi.org/10.1080/20008198.2017.1398003

Benyamini, Y., \& Solomon, Z. (2005). Combat stress reactions, posttraumatic stress disorder, cumulative life stress, and physical health among Israeli veterans twenty years after exposure to combat. Social Science \& Medicine, 61, 1267-1277. http://dx.doi.org/10.1016/j.socscimed.2005.01.023

Birkeland, M. S., \& Heir, T. (2017). Making connections: exploring the centrality of posttraumatic stress symptoms and covariates after a terrorist attack. European Journal 
PTSD and functional impairment

of Psychotraumatology, 8(sup3), 1333387.

http://dx.doi.org/10.1080/20008198.2017.1333387

Borsboom, D., \& Cramer, A.O.J. (2013). Network analysis: An integrative approach to the structure of psychopathology. Annual Review of Clinical Psychology, 9, 91-121. http://dx.doi.org/10.1146/annurev-clinpsy-050212-185608

Borsboom, D., Fried, E. I., Epskamp, S., Waldorp, L. J., van Borkulo, C. D., van der Maas, H. L. J., \& Cramer, A. O. J. (2017). False alarm? A comprehensive reanalysis of "Evidence that psychopathology symptom networks have limited replicability" by Forbes, Wright, Markon, and Krueger (2017). Journal of Abnormal Psychology, 126(7), 989-999. http://dx.doi.org/10.1037/abn0000306

Brewin, C. R., Andrews, B., \& Valentine, J. D. (2000). Meta-analysis of risk factors for posttraumatic stress disorder in trauma-exposed adults. Journal of Consulting and Clinical Psychology, 68(5), 748-766. http://dx.doi.org/10.1037/0022-006X.68.5.748

Choi, K. W., Batchelder, A. W., Ehlinger, P. P., Safren, S. A., \&O’Cleirigh, C. (2017). Applying network analysis to psychological comorbidity and health behaviour: Depression, PTSD, and sexual risk in sexual minority men with trauma histories. Journal of Consulting and Clinical Psychology, 85(12), 1158-1170. http://dx.doi.org/10.1037/ccp0000241

Chung, H., \& Breslau, N. (2008). The latent structure of post-traumatic stress disorder: tests of invariance by gender and trauma type. Psychological Medicine, 38(4), 563573. https://doi.org/10.1017/S0033291707002589

Cramer, A. O. J., Waldorp, L. J., van der Maas, H. L. J., \& Borsboom, D. (2010). Comorbidity: A network perspective. Behavioral and Brain Sciences, 33, 137-193. https://doi.org/10.1017/S0140525X09991567 
PTSD and functional impairment

Dobie, D. J., Kivlahan, D. R., Maynard, C., Bush, K. R., Davis, T. M., \& Bradley, K. A. (2004). Posttraumatic stress disorder in female veterans: Association with selfreported health problems and functional impairment. Archives of Internal Medicine, 164(4), 394-400. http://dx.doi.org/10.1001/archinte.164.4.394

Dorahy, M. J., \& van der Hart, O. (2015). DSM-5's posttraumatic stress disorder with dissociative symptoms: Challenges and future directions. Journal of Trauma \& Dissociation, 16, (1), 7-28. https://doi.org/10.1080/15299732.2014.908806

Epskamp, S., Borsboom, D. \& Fried, E.I. (2017). Estimating psychological networks and their accuracy: A tutorial paper. Behavior Research Methods. Advance online publication. https://doi.org/10.3758/s13428-017-0862-1

Epskamp, S., Cramer, A. O. J., Waldorp, L. J. Schmittmann, V. D., \&Borsboom, D. (2012). qgraph: Network visualizations of relationships in psychometric data. Journal of Statistical Software, 48(4), 1-18. http://doi.org/10.18637/jss.v048.i04

Epskamp, S., Waldorp, L. J., Mõttus, R., \& Borsboom, D. (2018). The Gaussian graphical model in cross-sectional and time-series data. Multivariate Behavioral Research. Advance online publication. https://doi.org/10.1080/00273171.2018.1454823

Fisher, A. J., Reeves, J. W., Lawyer, G., Medaglia, J. D., \& Rubel, J. A. (2017). Exploring the idiographic dynamics of mood and anxiety via network analysis. Journal of Abnormal Psychology, 126(8), 1044-1056. http://dx.doi.org/10.1037/abn0000311

Fletcher, S., Elklit, A., Shevlin, M., \& Armour, C. (2017). Predictors of PTSD treatment response trajectories in a sample of childhood sexual abuse survivors: The roles of social support, coping, and PTSD symptom clusters. Journal of Interpersonal Violence. Advance online publication. https://doi.org/10.1177/0886260517741212

Forbes, D., Lockwood, E., Elhai, J. D., Creamer, M., Bryant, R., McFarlane, A., ... O’Donnell, M. (2015). An evaluation of the DSM-5 factor structure for posttraumatic 
PTSD and functional impairment

stress disorder in survivors of traumatic injury. Journal of Anxiety Disorders, 29, 43-51. http://dx.doi.org/10.1016/j.janxdis.2014.11.004

Fried, E. I., Eidhof, M. B., Palic, S., Costantini, G., Huisman-van Dijk, H. M., Bockting, C.

L. H., ... Karstoft, K. I. (2018). Replicability and generalizability of posttraumatic stress disorder (PTSD) networks: A cross-cultural multisite study of PTSD symptoms in four trauma patient samples. Clinical Psychological Science. Advance online publication.https://doi.org/10.1177/2167702617745092

Friedman, J., Hastie, T., \&Tibshirani, R. (2008). Sparse inverse covariance estimation with the graphical lasso. Biostatistics, 9(3), 432-441. https://doi.org/10.1093/biostatistics/kxm045

Fruchterman, T. M. J., \&Reingold, E. M. (1991). Graph drawing by force-directed placement. Software: Practice and Experience, 21(11), 1129-1164.

Galatzer-Levy, I. R., \& Bryant, R. A. (2013). 636,120 ways to have posttraumatic stress disorder. Perspectives on Psychological Science, 8(6), 651-662. http://dx.doi.org/10.1177/1745691613504115

Galovski, T., \& Lyons, J. A. (2004). Psychological sequelae of combat violence: A review of the impact of PTSD on the veteran's family and possible interventions. Aggression and Violent Behavior, 9, 477-501. http://dx.doi.org/10.1016/S1359-1789(03)00045-4

Gewirtz, A. H., Polusny, M. A., DeGarmo, D. S., Khaylis, A., \&Erbes, C. R. (2010). Posttraumatic stress symptoms among national guard soldiers deployed to Iraq: Associations with parenting behaviors and couple adjustment. Journal of Consulting and Clinical Psychology, 78(5), 599-610. http://dx.doi.org/10.1037/a0020571

Hafstad, G. S., Dyb, G., Jensen, T. K., Steinberg, A. M., \&Pynoos, R. S. (2014). PTSD prevalence and symptom structure of DSM-5 criteria in adolescents and young adults 
PTSD and functional impairment

surviving the 2011 shooting in Norway. Journal of Affective Disorders, 169, 40-46. http://dx.doi.org/10.1016/j.jad.2014.06.055

Harvey, A. G. (2008). Insomnia, psychiatric disorders, and the transdiagnostic perspective. Current Directions in Psychological Science, 17(5), 299-303. https://doi.org/10.1111/j.1467-8721.2008.00594.x

Jones, P. (2018). networktools: Tools for identifying important nodes in networks. R package version 1.1.1. https://CRAN.R-project.org/package=networktools

Karstoft, K.I., Armour, C., Andersen, S. B., Bertelsen, M., \& Madsen, T. (2015). Community integration after deployment to Afghanistan: a longitudinal investigation of Danish soldiers. Social Psychiatry and Psychiatric Epidemiology, 50(4), 653-660. http://dx.doi.org/10.1007/s00127-014-0973-2

Kuhn, E., Blanchard, E. B., \& Hickling, E. J. (2003). Posttraumatic stress disorder and psychosocial functioning within two samples of MVA survivors. Behaviour Research and Therapy, 41, 1105-1112. http://dx.doi.org/10.1016/S0005-7967(03)00071-8

Lanius, R. A., Brand, B., Vermetten, E., Frewen, P. A., \& Spiegel, D. (2012). The dissociative subtype of posttraumatic stress disorder: Rationale, clinical and neurobiological evidence, and implications. Depression and Anxiety, 29, 701-708. http://dx.doi.org/10.1002/da.21889

Lunney, C. A., \&Schnurr, P. P. (2007). Domains of quality of life and symptoms in male veterans treated for posttraumatic stress disorder. Journal of Traumatic Stress, 20(6), 955-964. http://dx.doi.org/10.1002/jts.20269

Marks I. (1986). Behavioural psychotherapy. Bristol: John Wright, now published by I. Marks, Institute of Psychiatry, London.

McNally, R. J., Heeren, A., \& Robinaugh, D. J. (2017). A Bayesian network analysis of posttraumatic stress disorder symptoms in adults reporting childhood sexual abuse. 
PTSD and functional impairment

European Journal of Psychotraumatology, 8, 1341276.

http://dx.doi.org/10.1080/20008198.2017.1341276

McNally, R. J., Robinaugh, D. J., Wu, G. W. Y., Wang, L., Deserno, M. K., \&Borsboom, D. (2015). Mental disorders as causal systems: A network approach to posttraumatic stress disorder. Clinical Psychological Science, 3(6), 836-849.

http://dx.doi.org/10.1177/2167702614553230

Megías, J. L., Ryan, E., Vaquero, J. M. M., \&Frese, B. (2007). Comparisons of traumatic and positive memories in people with and without PTSD profile. Applied Cognitive Psychology, 21(1), 117-130.http://dx.doi.org/10.1002/acp.1282

Miller, M. W., Wolf, E. J., Kilpatrick, D., Resnick, H., Marx, B. P., Holowka, D. W., ... Friedman, R. C. (2013). The prevalence and latent structure of proposed DSM-5 posttraumatic stress disorder symptoms in U.S. national and veteran samples. Psychological Trauma: Theory, Research, Practice, and Policy, 5(6), 501-512. http://dx.doi.org/10.1037/a0029730

Mitchell, K. S., Wolf, E. J., Bovin, M. J., Lee, L. O., Green, J. D., Rosen, R. C., ... Marx, B. P. (2017). Network models of DSM-5 posttraumatic stress disorder: Implications for ICD-11. Journal of Abnormal Psychology, 126(3), 355-366.

http://dx.doi.org/10.1037/abn0000252

Murphy, D., Ashwick, R., Palmer, E., \& Busuttil, W. (2017). Describing the profile of a population of UK veterans seeking support for mental health difficulties. Journal of Mental Health. Advance online publication. http://dx.doi.org/10.1080/09638237.2017.1385739

Murphy, D., Ross, J., Ashwick, R., Armour, C., \& Busuttil, W. (2017). Exploring optimum cut-off scores to screen for probable posttraumatic stress disorder within a sample of 
PTSD and functional impairment

UK treatment-seeking veterans. European Journal of Psychotraumatology, 8(1), 1398001. https://doi.org/10.1080/20008198.2017.1398001

North, C. S., Nixon, S. J., Shariat, S., Mallonee, S., McMillen, C., Spitznagel, E. L., \& Smith, E. M. (1999). Survivors of the Oklahoma City bombing. JAMA, 282(8), 755-762. https://doi.org/10.1001/jama.282.8.755

Opsahl, T., Agneessens, F., \&Skvoretz, J. (2010). Node centrality in weighted networks: Generalizing degree and shortest paths. Social Networks, 32(3), 245-251. https://doi.org/10.1016/j.socnet.2010.03.006

Pietrzak, R. H., Feder, A., Schechter, C. B., Singh, R., Cancelmo, L., Bromet, E. J., ... Southwick, S. M. (2014). Dimensional structure and course of post-traumatic stress symptomatology in World Trade Center responders. Psychological Medicine, 44(10), 2085-2098. http://dx.doi.org/10.1017/S0033291713002924

Pietrzak, R. H., Tsai, J., Armour, C., Mota, N., Harpaz-Rotem, I., \& Southwick, S. M. (2015). Functional significance of a novel 7-factor model of DSM-5 PTSD symptoms: Results from the National Health and Resilience in Veterans Study. Journal of Affective Disorders, 174, 522-526. http://dx.doi.org/10.1016/j.jad.2014.12.007

Pittman, J. O., Goldsmith, A. A., Lemmer, J. A., Kilmer, M. T., \& Baker, D. G. (2012). Posttraumatic stress disorder, depression, and health-related quality of life in OEF/OIF veterans. Quality of Life Research, 21(1), 99-103. http://dx.doi.org/10.1007/s11136011-9918-3

Porter, S., \&Birt, A. R. (2001). Is traumatic memory special? A comparison of traumatic memory characteristics with memory for other emotional life experiences. Applied Cognitive Psychology, 15, S101-S117. https://doi.org/10.1002/acp.766

Price, M., \& van Stolk-Cooke, K. (2015). Examination of the interrelations between the factors of PTSD, major depression, and generalized anxiety disorder in a heterogeneous 
PTSD and functional impairment

trauma-exposed sample using DSM-5 criteria. Journal of Affective Disorders, 186, 149155. https://doi.org/10.1016/j.jad.2015.06.012

Roley, M. E., Contractor, A. A., Weiss, N. H., Armour, C., \& Elhai, J. D. (2017). Impulsivity Facets' Predictive Relations with DSM-5 PTSD Symptom Clusters. Psychological Trauma: Theory, Research, Practice, and Policy, 9(1), 76-79. http://dx.doi.org/10.1037/tra0000146

Riggs, D. S., Byrne, C. A., Weathers, F. W., \&Litz, B. T. (1998). The quality of the intimate relationships of male Vietnam veterans: Problems associated with posttraumatic stress disorder. Journal of Traumatic Stress, 11(1), 87-101. http://dx.doi.org/10.1023/A:1024409200155

Rodriguez, P., Holowka, D. W., \& Marx, B. P. (2012). Assessment of posttraumatic stress disorder-related functional impairment: a review. Journal of Rehabilitation Research and Development, 49(5), 649-665. http://dx.doi.org/10.1682/JRRD.2011.09.0162

Rona, R. J., Jones, M., Iversen, A., Hull, L., Greenberg, N., Fear, N. T., ... Wessely, S. (2009). The impact of posttraumatic stress disorder on impairment in the UK military at the time of the Iraq war. Journal of Psychiatric Research, 43, 649-655. http://dx.doi.org/10.1016/j.jpsychires.2008.09.006

Ross, J., Baník, G., Dědová, M., Mikulášková, G., \& Armour, C. (2018). Assessing the structure and meaningfulness of the dissociative subtype of PTSD. Social Psychiatry and Psychiatric Epidemiology, 53, 87-97. https://doi.org/10.1007/s00127-017-1445-2

Ruscio, A. M., Weathers, F. W., King, L. A., \& King, D. W. (2002). Male war-zone veterans' perceived relationships with their children: The importance of emotional numbing. Journal of Traumatic Stress, 15(5), 351-357. http://dx.doi.org/10.1023/A:1020125006371 
PTSD and functional impairment

Sayer, N. A., Orazem, R. J., Noorbaloochi, S., Gravely, A., Frazier, P., Carlson, K. F., ... Oleson, H. (2015). Iraq and Afghanistan war veterans with reintegration problems: Differences by Veterans Affairs healthcare user status. Administration and Policy in Mental Health, 42(4), 493-503. http://dx.doi.org/10.1007/s10488-014-0564-2

Schnurr, P. P., Lunney, C. A. (2008). Exploration of gender differences in how quality of life relates to posttraumatic stress disorder in male and female veterans. Journal of Rehabilitation Research \& Development, 45(3), 383-394. https://doi.org/10.1682/JRRD.2007.06.0099

Schnurr, P. P., Lunney, C. A., Bovin, M. J., \& Marx, B. P. (2009). Posttraumatic stress disorder and quality of life: Extension of findings to veterans of the wars in Iraq and Afghanistan. Clinical Psychology Review, 29, 727-735. https://doi.org/10.1016/j.cpr.2009.08.006

Seligowski, A. V., \& Orcutt, H. K. (2016). Support for the 7-factor hybrid model of PTSD in a community sample. Psychological Trauma: Theory, Research, Practice, and Policy, 8(2), 218-221. https://doi.org/10.1037/tra0000104

Shea, M. T., Vujanovic, A. A., Mansfield, A. K., Sevin, E., \& Liu, F. (2005). Posttraumatic stress disorder symptoms and functional impairment among OEF and OIF national guard and reserve veterans. Journal of Traumatic Stress, 23(1), 100-107. http://dx.doi.org/10.1002/jts.20497

Smith, M. W., Schnurr, P. P., \&Rosenheck, R. A. (2005). Employment outcomes and PTSD symptom severity. Mental Health Services Research, 7(2), 89-101.

Spiller, T. R., Schick, M., Schnyder, U., Bryant, R. A., Nickerson, A., \& Morina, N. (2017). Symptoms of posttraumatic stress disorder in a clinical sample of refugees: a network analysis. European Journal of Psychotraumatology, 8(sup3), 1318032. https://doi.org/10.1080/20008198.2017.1318032 
PTSD and functional impairment

Taft, C. T., Watkins, L. E., Stafford, J., Street, A. E., \& Monson, C. M. (2011). Posttraumatic stress disorder and intimate relationship problems: A meta-analysis. Journal of Consulting and Clinical Psychology, 79(1), 22-33. http://dx.doi.org/10.1037/a0022196

Terluin, B., de Boer, M. R., \& de Vet H. C. W. (2016). Difference sin connection strength between mental symptoms might be explained by differences in variance: Reanalysis of network data did not confirm staging. PLoS ONE, 11(11), e0155205. http://dx.doi.org/10.1371/journal.pone.0155205

Van Borkulo, C., Boschloo, L., Borsboom, D., Penninx, B. W. J. H., Waldorp, L. J., \&Schoevers, R. A. (2015). Association of symptom network structure with the course of depression. JAMA Psychiatry, 72(12), 1219-1226. http://dx.doi.org/10.1001/jamapsychiatry.2015.2079

Von Stockert, S. H. H., Fried, E. I., Armour, C., \& Pietrzak, R. H. (2018). Evaluating the stability of DSM-5 PTSD symptom network structure in a national sample of U.S. military veterans. Journal of Affective Disorders, 229, 63-68. https://doi.org/10.1016/j.jad.2017.12.043

Weathers, F.W., Litz, B.T., Keane, T.M., Palmieri, P.A., Marx, B.P., \&Schnurr, P.P. (2013). The PTSD Checklist for DSM-5 (PCL-5). Scale available from the National Center for PTSD at www.ptsd.va.gov

Wisco, B. E., Marx, B. P., Wolf, E. J., Miller, M. W., Southwick, S. M., \& Pietrzak, R. H. (2014). Posttraumatic stress disorder in the US veteran population: Results from the National Health and Resilience in Veterans Study. Journal of Clinical Psychiatry, 75(12), 1338-1346. http://dx.doi.org/10.4088/JCP.14m09328

World Health Organization (2018). ICD-11 Beta Draft (Mortality and Morbidity Statistics). Retrieved February 7, 2018, from https://icd.who.int/dev11/1-m/en 
PTSD and functional impairment

Wright, K. M., Cabrera, O. A., Eckford, R. D., Adler, A. B., \&Bliese, P. D. (2012). The impact of predeployment functional impairment on mental health after combat. Psychological Trauma: Theory, Research, Practice, and Policy, 4(3), 260-269. http://dx.doi.org/10.1037/a0024373

Yufik, T., \& Simms, L. J. (2010). A meta-analytic investigation of the structure of posttraumatic stress disorder symptoms. Journal of Abnormal Psychology, 119(4), 764776. https://doi.org/10.1037/a0020981 
PTSD and functional impairment

Figure 1. Regularized partial correlation network of the 20 PTSD symptoms

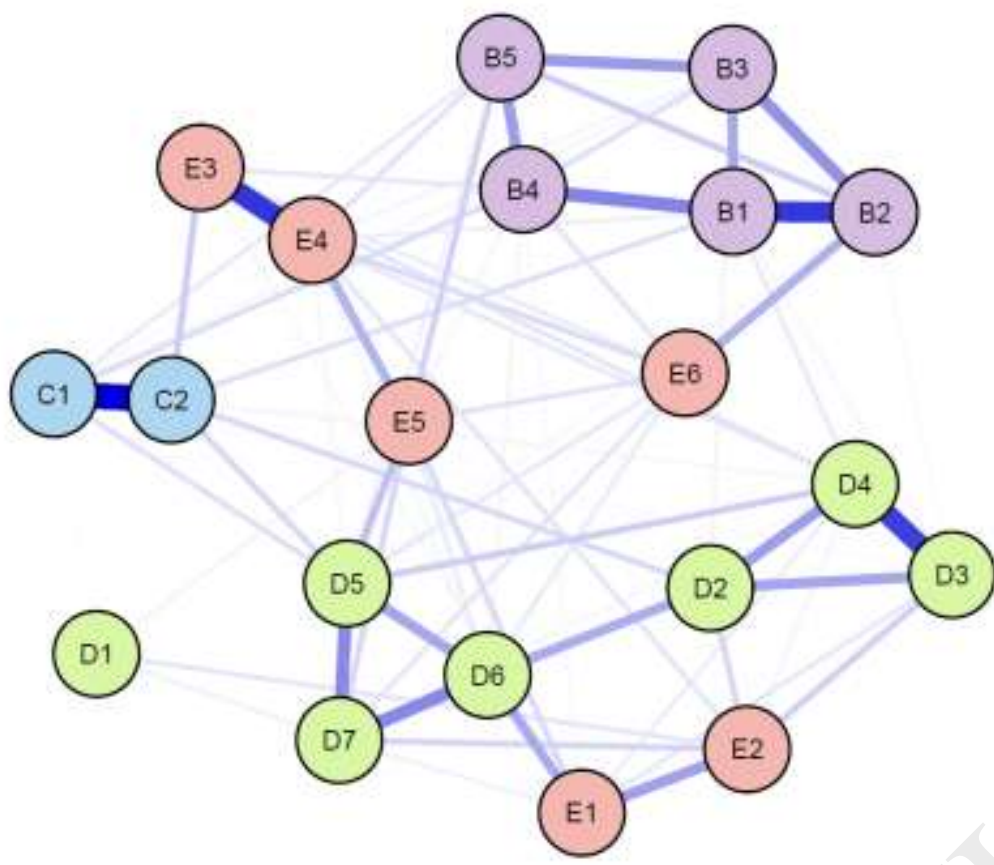

Re-experiencing

- B1: Recurrent thoughts

- B2: Nightmares

- B3: Flashbacks

- B4: Psychological cue reactivity

- B5: Physiological cue reactivity

Avoidance

C1: Avoodance of thoughts

- C2- Avoidance of reminders

Negative alterations in cognition and mood - D1: Memory impairment

D2: Negative beliefs

- D3: Distorted blame

D4: Negative emotional state

- D5: Diminished interest

D6: Dotachment

D7: Restricted affect

Alterations in arousal and reactivity

- E1: Irritability or anger

- E2: Reckless or self-destructive behaviour

- E3: Hypervigilance

E4: Exaggerated startle

E5: Difficulty concentrating

E6: Sleeping difficulties 
Running head: PTSD and functional impairment

Figure 2. Standardized node centrality estimates of the 20-symptom PTSD network
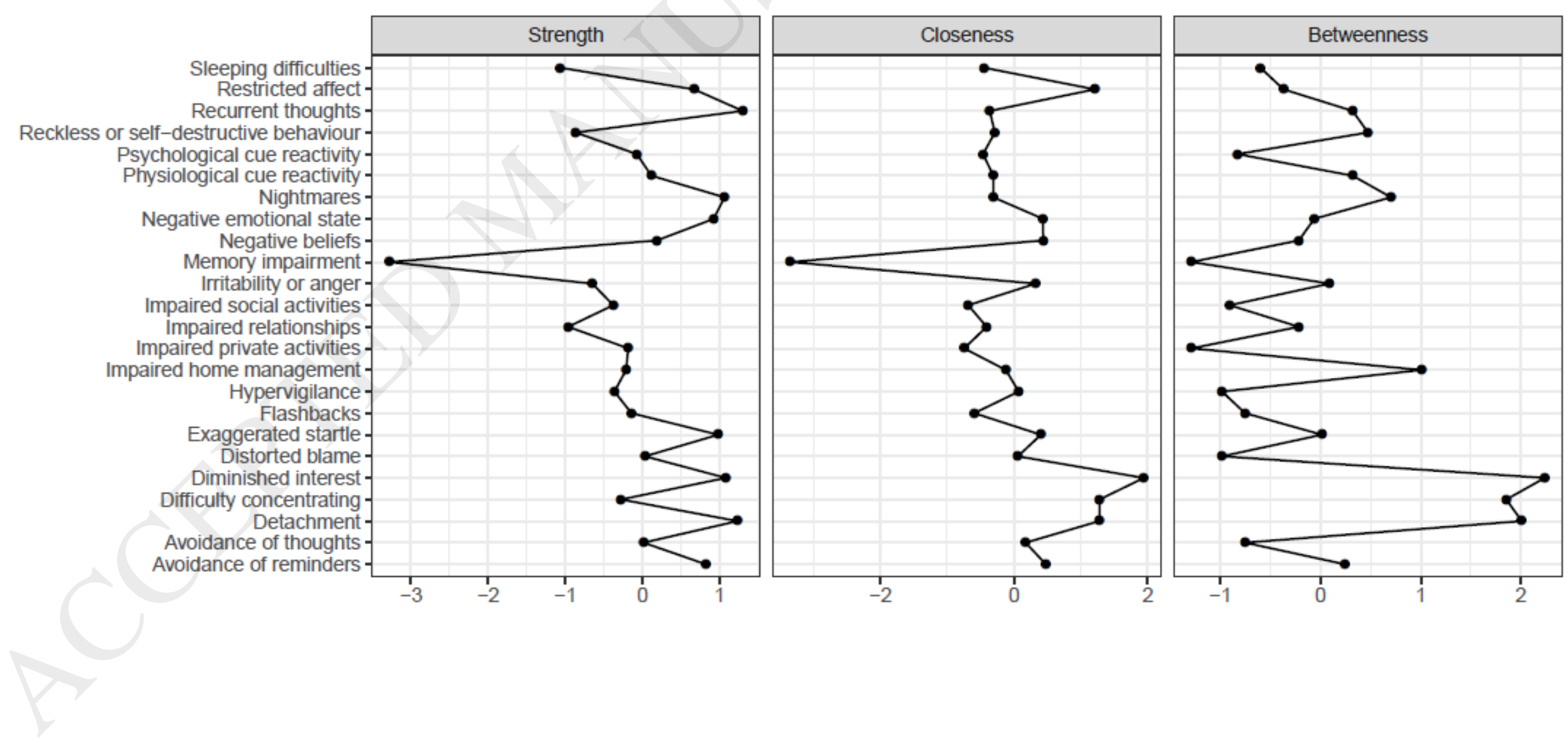
Running head: PTSD and functional impairment

Figure 3. Regularized partial correlation network of the 20 PTSD symptoms and four functional impairment items

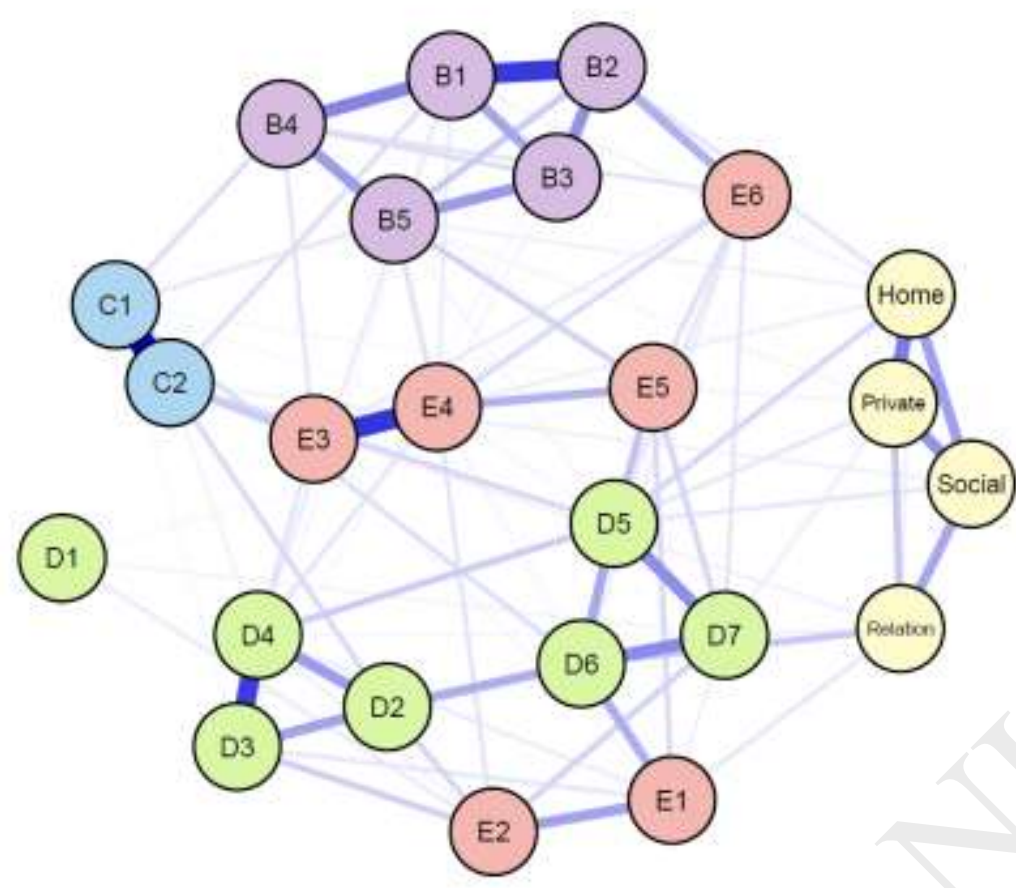

Functional impairment

- Home: Impaired home management

- Social: Impaired social activities

- Private: Impaired private activities

- Relation: Impaired relationships

Re-experiencing

- B1: Recurrent thoughts.

- B2: Nightmares

- B3: Flashbacks

- B4: Psychological cue reactivity

- 85: Physiological cue reactivity

Avoidance

o C1: Avoidance of thoughts

- C2: Avoidance of reminders

Negative alterations in cognition and mood

- D1: Memory impairment

- D2: Negative beliefs

D3: Distorted blame

- D4: Negative emotional state

- D5: Diminished interest

- D6: Detachment

- D7: Restricted affect

Alterations in arousal and reactivity

- E1: Irritability or anger

- E2 Reckless or self-destructive behaviour

- E3: Hypervigilance

- E4: Exaggerated startle

- E5: Difficulty concentrating

- E6: Sleeping difficulties 
Running head: PTSD and functional impairment

Figure 4. Standardized bridge centrality estimates in the PTSD with functional impairment network
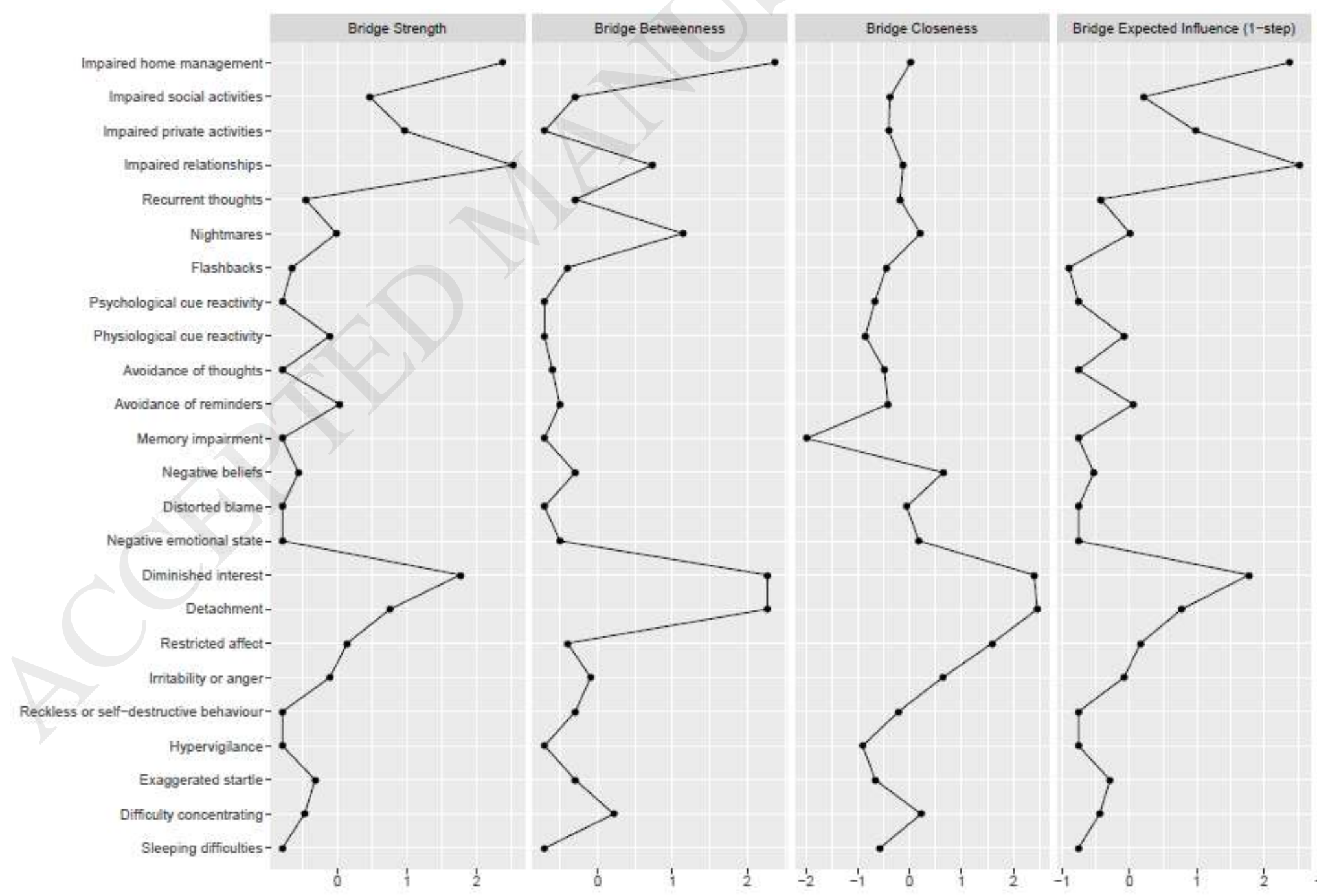

Bridge Expected Influence (2-step)
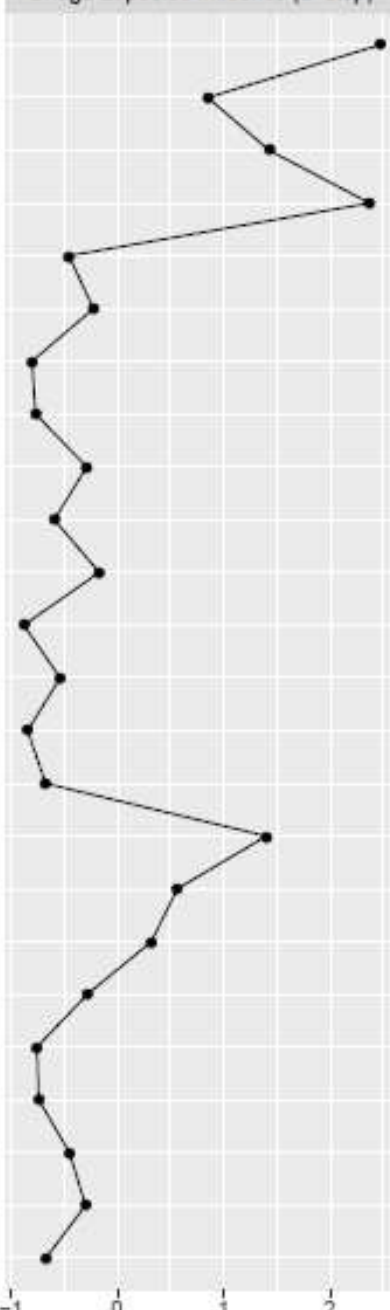